\title{
Quantum Probabilities versus Event Frequencies
}

\author{
Charis Anastopoulos \\ Department of Physics, University of Patras, 26500 Patras, Greece
}

Received on 19 December, 2004

\begin{abstract}
Quantum probability is very different from classical probability. Part of this difference is manifested in the generic inability of stochastic processes to describe the results of multi-time measurements of quantum mechanical systems and the fact that the complex-valued temporal correlation functions of quantum theory have no interpretation in terms of multi-time measurements. By analysing experiments involving measurements at more than one moments of time, we conclude that this inequivalence must be manifested either as a failure of the quantum logic or as the inability to define probabilities in multi-time measurements because the relative frequencies do not converge. These alternatives can be empirically distinguished as they correspond to different behaviours of the statistical data in multi-time measurements.
\end{abstract}

\section{Introduction}

Quantum mechanics is a probabilistic theory; its physical predictions are phrased in terms of probabilities. As such any experiment testing the quantum mechanical predictions needs to make some assumptions about the relation of probabilities-in the abstract mathematical formalism of quantum mechanicsto events that are actually being measured. For that purpose one invariably employs the relation of probabilities to event frequencies, namely we consider that the number provided by the rules of quantum theory as the probability $p(C)$ of an event $C$, the number of times $C$ is realised after $n$ trials, divided by $n$, when the limit $n \rightarrow \infty$ is taken. Clearly, the limit $n \rightarrow \infty$ is not something that can be physically achieved, and one assumes a relatively rapid convergence of the relative frequencies.

More concretely, the relation of probabilities to event frequencies may be formalised as follows. We assume an ensemble of a large number of identically prepared systems. In each system we measure some physical properties say $A$, which take value in a set $\Omega$. We then perform the measurements one by one - thus constructing a sequence $A_{n}$ of points of $\Omega$, where $n$ is an integer that labels the experiments. We next sample the measurement outcomes into subsets $U$ of $\Omega$.

We next define $\nu(U, n)$ as the number of times that the result of the measurement is found in $U$ in the first $n$ experiments. It is evident that $\nu(U, n)$ satisfies the following properties

$$
\begin{aligned}
\nu(U, n) & \geq \nu(U, m), \text { if } n>m \\
\nu(U \cup V, n) & =\nu(U, n)+\nu(V, n), \text { if } U \cap V=\emptyset \\
\nu(\Omega, n) & =n \\
\nu(\emptyset, n) & =0
\end{aligned}
$$

One then may define the probability that an event in $U$ has been realised as

$$
p(U)=\lim _{n \rightarrow \infty} \frac{\nu(U, n)}{n},
$$

provided the limit exists.

The definition above of probabilities is compatible with the mean value of the observable $A$ as

$$
\langle A\rangle=\lim _{n \rightarrow \infty} \frac{1}{n} \sum_{i=1}^{n} A_{i}
$$

and similarly for higher moments of the observable $A$.

The relation of probabilities to event frequencies is not a feature of quantum probabilities alone. It is a basic assumption about the relation of the probabilistic modelling of a physical system to the concrete experimental date one obtains from the study of such systems. It is valid, whether the probability theory that provides the model of the system is classical or quantum.

The relation of probabilities to event frequencies is not valid only for measurements of a single variable at one moment of time. The sample space $\Omega$ may correspond to measurements of a single system at two consecutive moments of time-for example measuring the position of a particle at time $t_{1}$ and at time $t_{2}$. In that case $\Omega=\Omega_{t_{1}} \times \Omega_{t_{2}}$, where $\Omega_{t_{i}}$ is the sample space for the single-time measurements at time $t_{i}$. The outcome of a single measurement is a Cartesian pair of values $\left(A_{t_{1}}, A_{t_{2}}\right)_{n}$, where $n$ again labels the experimental run. From this sequence, one may construct the sequence $\nu\left(U_{1}, t_{1} ; U_{2}, t_{2} ; n\right)$ the particle's position was found within $U_{1}$ at time $t_{1}$ and within $U_{2}$ at time $t_{2}$ in $n$ runs of the experiment, we obtain the corresponding probability by Eq. (5). Alternatively, one may define the two-time correlation function as

$$
\left\langle A_{t_{1}} A_{t_{2}}\right\rangle=\lim _{n \rightarrow \infty} \frac{1}{n^{2}} \sum_{i=1}^{n}\left[\left(A_{t_{1}}\right)_{i}-\left\langle A_{t_{1}}\right\rangle\right]\left[\left(A_{t_{2}}\right)_{i}-\left\langle A_{t_{2}}\right\rangle\right]
$$

In classical probability theory the multi-time probabilities can be obtained from a stochastic process-through the definition of a probability measure $d \mu$ in the space of all histories 
$A(\cdot)$ of the variable $A$. The two-time correlation function is then expressed as

$$
\left\langle A_{t_{1}} A_{t_{2}}\right\rangle=\int d \mu[A(\cdot)] A\left(t_{1}\right) A\left(t_{2}\right)
$$

But an equation like (8) is in general not possible in quantum theory. The statistical properties of quantum measurements at more than one moments of time cannot be modelled by a stochastic process. This is essentially a generalisation of Bell' $s$ theorem: a stochastic process may at most provide is the mean values of a single observable at all moments of time. This is the case, for instance, for Nelson's stochastic mechanics [1]. The higher order correlation functions predicted by quantum theory cannot be described by a stochastic process.

But this inequivalence between quantum theory and classical stochastic processes does not end here. The problem is that the quantum mechanical correlation functions are, in general, complex valued, rather than real-valued. This means that they cannot have an interpretation in terms of event frequencies as in Eq. (7), which always leads to real-valued correlation functions. The question then arises about the physical interpretation of the quantum correlation functions, but also the inverse. Suppose we perform a two-time measurement on a quantum system, obtain a sequence $\left(A_{t_{1}}, A_{t_{2}}\right)_{n}$ of measurement outcomes and construct the two-point correlation function (7) from the experimental data. How is such a correlation function theoretically predicted from the standard quantum mechanical formalism? It equals the quantum mechanical correlation function, only if the latter is real-valued. This is only possible, if the self-adjoint operator corresponding to the observable $A$ commutes with the Hamiltonian.

\section{Probabilities in two-time measure- ments}

To address the issues raised above, we consider a two-time quantum measurement of position. We assume a particle source, which can be controlled so finely as to emit a single particle at a time. Two thin sheets of penetrable material are placed one after the other in front of the particle source, both parallel to the $\mathrm{x}-\mathrm{y}$ plane. Particles leave tracks as they cross through the sheets, and one may then determine their $x$ and $y$ coordinates. We assume that the particles are prepared in a state sharply concentrated in momentum around a specific value $p_{z}$ in the direction normal to the detectors. For example, the $z$-degrees of freedom may be represented by the wave function

$$
\psi(z)=\frac{1}{\left(2 \pi \sigma_{z}\right)^{1 / 4}} e^{-\frac{z^{2}}{4 \sigma_{z}^{2}}+i p_{z} z}
$$

such that the spread $\Delta p_{z}=1 / \sigma_{z}<<p_{z}$. If $\delta p_{z}$ is the momentum-transfer as the particle crosses the first detector the relative error in the determination of the time-of-arrival is $\frac{\delta t}{t_{2}-t_{1}}=\frac{\delta p_{z}+\Delta p_{z}}{p_{z}}$, which can be made sufficiently small for large values of $p_{z}$. Consequently, the recordings of each sheet correspond to measurements at specific moments of time $t_{1}$ and $t_{2}$-predetermined by the source's energy and the placement of the sheets.

As we described earlier each time the source emits a particle we record the readings $\left(x_{1}, t_{1} ; x_{2}, t_{2}\right)_{n} ; n$ labels the experimental runs and the $y$ coordinate is suppressed for brevity. We thus construct a sequence of measurement outcomes. From this one may define the sequence $\nu_{n}\left(U_{1}, t_{2} ; U_{2}, t_{2}\right)$ for each pair of regions $U_{1}$ of the sheet at $t_{1}$ and $U_{2}$ of the sheet at $t_{2}: \nu_{n}\left(U_{1}, t_{2} ; U_{2}, t_{2}\right)$ is defined as the number of times within the first $n$ measurements that $x_{1} \in U_{1}$ and $x_{2} \in U_{2}$. From the sequence $\nu_{n}$ one may obtain the probability $p\left(U_{1}, t_{1} ; U_{n}, t_{n}\right)$ as the limit $\nu_{n}\left(U_{1}, t_{1} ; U_{n}, t_{n}\right) / n$ as $n \rightarrow \infty$.

The rules of quantum theory allow us to express this probability in terms of the projection operators $\hat{P}_{i}$ that correspond to the interval $U_{i}$ of the particle's position.

$$
p\left(U_{i}, t_{1} ; U_{j}, t_{2}\right)=\operatorname{Tr}\left(\hat{Q}_{j} \hat{P}_{i} \rho\left(t_{1}\right) \hat{P}_{i}\right),
$$

where we denoted for simplicity

$$
\hat{Q}_{j}=e^{i \hat{H}\left(t_{2}-t_{1}\right)} \hat{P}_{j} e^{-i \hat{H}\left(t_{2}-t_{1}\right)} .
$$

$\hat{H}$ the Hamiltonian of the particle and $\rho\left(t_{1}\right)$ the initial density matrix evolved until time $t_{1}$.

Suppose, however, we consider the probability that the particle first crossed through either $U_{1}$ or $U_{2}$ and then through $U_{j}$. The projection operator corresponding to $U_{1} \cup U_{2}$ is $\hat{P}_{1}+\hat{P}_{2}$, hence the corresponding probability is

$$
\begin{aligned}
& p\left(U_{1} \cup U_{2}, t_{1} ; U_{j}, t_{2}\right)= \\
& \operatorname{Tr}\left(\hat{Q}_{j}\left(\hat{P}_{1}+\hat{P}_{2}\right) \hat{\rho}\left(t_{1}\right)\left(\hat{P}_{1}+\hat{P}_{2}\right)\right) \\
& =p\left(U_{1}, t_{1} ; U_{j}, t_{2}\right)+p\left(U_{2}, t_{1} ; U_{j}, t_{2}\right) \\
& +2 \operatorname{Red}\left(U_{1}, U_{2}, t_{1} ; U_{j}, t_{2}\right),
\end{aligned}
$$

where

$$
d\left(U_{1}, U_{2}, t_{1} ; U_{j}, t_{2}\right)=\operatorname{Tr}\left(\hat{Q}_{j} \hat{P}_{1} \hat{\rho}\left(t_{1}\right) \hat{P}_{2}\right)
$$

is known as the decoherence functional in the consistent histories approach $[2,3,4,5]$.

On the other hand the elementary properties of the frequencies state that $\nu\left(\left[U_{1} \cup U_{2}\right] \times U_{j}, n\right)=\nu\left(U_{1} \times U_{j}, n\right)+$ $\nu\left(U_{2} \times U_{j}, n\right)$, so that in the limit $n \rightarrow \infty$

$$
\begin{aligned}
& p\left(U_{1} \cup U_{2}, t_{1} ; U_{j}, t_{2}\right)= \\
& \quad p\left(U_{1}, t_{1} ;, U_{j}, t_{2}\right)+p\left(U_{2}, t_{1} ; U_{j}, t_{2}\right)
\end{aligned}
$$

In other words, the quantum mechanical probabilities are not additive (unless the consistency condition $\operatorname{Red}\left(U_{1}, U_{2}, t_{1} ; U_{j}, t_{2}\right)=0$ is satisfied), while the measured frequencies of events are additive. There seems to be a conflict between the natural predictions of the quantum mechanical formalism and the usual relation of probabilities to event frequencies that is employed in the interpretation of experimental data. To resolve this conflict we must construct probabilities for the two-time measurements without abandoning the standard rules of quantum theory. The problem is that we do not know a priori if the probabilities $p\left(U_{1}, t_{1} ; U_{2}, t_{2}\right)$ can actually be defined from the experimental data; namely if the 
relative frequencies converge or not. This issue has not been settled empirically, because no precision experiment has yet been designed with that issue in mind. The rules of quantum theory may account for both eventualities. But since the physical behaviour is different, the resulting statistical predictions will also be different.

\section{First alternative: frequencies con- verge}

The first alternative involves the assumption that probabilities for multi-time measurements actually exist. We must sacrifice something if such definition is possible-for otherwise, these probabilities could be used to construct a stochastic process that reproduces all predictions of quantum theory. It turns out that in that case one has to sacrifice the logical structure of quantum mechanical measurements. To see this, we must identify the erroneous assumption in the derivation of equation (10) [6].

We start from a density matrix $\hat{\rho}$ at $t=0$, which is evolved unitarily until time $t_{1}$, when the particle enters the measuring device. If we register the particle in the interval labelled by $i$, the outcoming density matrix equals

$$
\frac{\hat{P}_{i} \hat{\rho}\left(t_{1}\right) \hat{P}_{i}}{\operatorname{Tr}\left(\hat{\rho}\left(t_{1}\right) \hat{P}_{i}\right)}
$$

We make no commitments about the interpretation of the measurement process. It is irrelevant whether the measuring device is classical like in Copenhagen quantum theory, or quantum mechanical and a physical process of wave packet reduction has taken place. It also makes little difference whether the density matrix refers to an individual system, or a statistical ensemble, because at the end of the day our results will be interpreted by statistical processing of the measurement outcomes. What is important is that the density matrix (15) allows us to compute the conditional probabilities that the event $j$ takes place at $t_{2}$ provided the event $i$ took place at $t_{1}$

$$
\frac{\operatorname{Tr}\left(\hat{P}_{i} \hat{\rho}\left(t_{1}\right) \hat{P}_{i} \hat{Q}_{j}\right)}{\operatorname{Tr}\left(\hat{\rho}\left(t_{1}\right) \hat{P}_{i}\right)}
$$

from which the classical definition of conditional probability leads us to expression (10) for the probability that first the event $i$ takes place at $t_{1}$ and then the event $j$ takes place at time $t_{2}$.

The problem lies in equation (15). If, instead of sampling the measurement outcomes in the set, say $U_{1}$, we sampled it into $U_{1} \cup U_{2}$, we would have employed the projector $\hat{P}_{1}+\hat{P}_{2}$ and the out-coming density matrix would equal

$$
\frac{\left(\hat{P}_{1}+\hat{P}_{2}\right) \hat{\rho}\left(t_{1}\right)\left(\hat{P}_{1}+\hat{P}_{2}\right)}{\left.\operatorname{Tr}\left(\hat{\rho}\left(t_{1}\right)\left(\hat{P}_{1}+\hat{P}_{2}\right)\right)\right)}
$$

We would then obtain the result (10), which is inconsistent with the probabilities defined through relative frequency.
However, there is no a priori reason to use equation (15) for the out-coming density matrix. The action of the projection $\hat{P}_{i}$ depends on our choice of sampling of measurement outcomes and not on the measurement outcome itself. What has actually taken place is that the particle left a mark on a specific point, and we then choose to place that point into one or the other set. If we had a measurement at a single moment of time, this would not have been a problem, because the density matrix (15) does not appear in any physical predictions for single-time measurements. In a single-time measurement the only physically relevant quantities are the probabilities $\operatorname{Tr}\left(\hat{P}_{i} \hat{\rho}\left(t_{1}\right)\right)$, which are additive and for this reason they do not depend on our choice of sampling.

In the two-time measurement, however, the probabilities turn out to be non-additive. We should therefore be very cautious in any use of conditional probability. If we sample events into larger sets than the ones being manifested in the experiments, then we employ less information than what we have actually obtained. Our use of conditional probabilities will be, therefore, improper.

The physically correct procedure would be to incorporate in our probabilities all information that has been obtained from the measurements. In other words we must construct the out-coming density matrix not on the basis of our arbitrary choice of sampling events, but on what we have actually observed. We should not use an arbitrary set of projectors, but only the finest possible projectors compatible with the resolution of the apparatus. If $\delta$ is the sharpest resolution of the measuring device (say the width of the dots indicating the particle's position) the relevant projectors are $\hat{P}_{x}^{\delta}$, which project onto the interval $\left[x-\frac{\delta}{2}, x+\frac{\delta}{2}\right]$. Using these projectors we construct the probabilities

$$
\begin{aligned}
& p_{\delta}\left(x_{1}, t_{1} ; x_{2}, t_{2}\right)= \\
& \quad \operatorname{Tr}\left(e^{i \hat{H}\left(t_{2}-t_{1}\right)} \hat{P}_{x_{2}}^{\delta} e^{-i \hat{H}\left(t_{2}-t_{1}\right)} \hat{P}_{x_{1}}^{\delta} \hat{\rho}\left(t_{1}\right) \hat{P}_{x_{1}}^{\delta}\right),
\end{aligned}
$$

that a dot will be found centered at the point $x_{1}$ in the first measurement and then a dot centered at the point $x_{2}$ in the second measurement.

We may then construct the probabilities for a particle to be found within a subset $U_{i}$ of $\mathbf{R}$ at time $t_{1}$ and then within a subset $U_{j}$ at time $t_{2}$. For this purpose, we split each set $U_{i}$ into mutually exclusive cells $u_{\alpha i}$ of size $\delta$, such that

$$
\begin{aligned}
\cup_{\alpha} u_{\alpha i} & =U_{i} \\
u_{\alpha i} \cap u_{\beta i} & =\emptyset, \alpha \neq \beta .
\end{aligned}
$$

If we denote select points $x_{\alpha i} \in u_{\alpha i}$, for all $i$ ( $x_{\alpha i}$ may be the midpoint of $u_{\alpha i}$ ), we may construct the probability $p_{\delta}\left(U_{i}, t_{1} ; U_{j}, t_{2}\right)$

$$
p_{\delta}\left(U_{i}, t_{1} ; U_{j}, t_{2}\right)=\sum_{\alpha} \sum_{\beta} p_{\delta}\left(x_{\alpha i}, t_{1} ; x_{\beta j}, t_{2}\right)
$$

In the limit that the typical size of the sets $U_{j}$ is much larger than $\delta$, we may approximate the summation by an integral,

$p_{\delta}\left(U_{i}, t_{1} \mid U_{j}, t_{2}\right)=\frac{1}{\delta^{2}} \int_{U_{i}} d x_{1} \int_{U_{j}} d x_{2} p_{\delta}\left(x_{1}, t_{1} ; x_{2}, t_{2}\right)$ 
In other words, the objects $\frac{1}{\delta^{2}} p_{\delta}\left(U_{i}, t_{1} \mid U_{j}, t_{2}\right)$ play the role of probability densities. The probabilities (22) are compatible with the relative frequencies, because they do satisfy the additivity criterion.

The problem is that the dependence of the probabilities $p_{\delta}\left(U_{i}, t_{1} \mid U_{j}, t_{2}\right)$ in Eq. (22) on the sample sets $U_{1}$ and $U_{2}$ is not through the projectors $\hat{P}_{U_{1}}, \hat{P}_{U_{2}}$. Instead, they depend strongly on $\delta$, which depends itself on the physical properties of the measuring device as well as the specific characteristics of the initial state. This dependence is not as benign as its counterpart in classical probability theory- the measured probabilities do not converge to some ideal value, when the size $d$ is taken very small. If we double our accuracy ( $\delta \rightarrow \delta / 2$ ), we do not obtain a better estimate of the probabilities, but rather a very different probability assignment for all events. To see this we estimate the difference

$$
\epsilon_{\delta}\left(U_{1}, t_{1} \mid U_{2}, t_{2}\right)=p_{\delta}\left(U_{1}, t_{1} \mid U_{2}, t_{2}\right)-p_{\frac{\delta}{2}}\left(U_{1}, t_{1} \mid U_{2}, t_{2}\right)
$$

in the probabilities for two successive outcomes $\left(U_{i}, U_{j}\right)$, for measurement devices characterised by values $\delta$ and $\frac{\delta}{2}$ for the resolution. Employing equation (22) we may estimate [6] that for a free particle with mass $m$

$$
\epsilon_{\delta}\left(U_{1}, t_{1} ; U_{2}, t_{2}\right)=c p_{\delta}\left(U_{1}, t_{1} ; U_{2}, t_{2}\right) \cos \left(P \delta+\frac{b \Delta}{\delta}\right),
$$

where $c$ is a number of the order of unity, $P$ is the mean momentum of the initial state, $b$ is a function of $t=t_{2}-t_{1}, \delta$ and $m . \Delta=X_{2}-X_{1}-P / m t$, and $X_{1}, X_{2}$ are the center points of the sets $U_{1}, U_{2}$ respectively. It is clear from Eq. (24) that the difference in probabilities is of the same order of magnitude as the probabilities themseleves for all values of $\delta$ and there exists generically no convergence in probabilities as the resolution becomes smaller. We do not get better results when we improve the resolution, we only get different results.

The property above of the single-time quantum probabilities has as consequence that all physical measurements may be described in terms of YES-NO experiments [7]. A typical YES-NO experiment involves a slit on a curtain placed in front of a particle beam and a detector behind the slit measuring whether the particle crossed through the slit or not. The slit is represented mathematically by a projection operator in the range of position. After a large number of trials $(n)$ we may determine the probability that the particle crossed through the slit as the number of times the detector register a particle divided by the $n$. If we repeat this experiment with different filters that correspond to the same physical property (the associated projectors commute), we may eventually reconstruct the probability distribution for this quantity, because the single time probabilities are additive. Hence, the probabilities $\operatorname{Tr}\left(\hat{\rho} \hat{P}_{U}\right)$ determined by the YES-NO measurement of position in the set $U$ coincide with the probabilities determined through event frequencies in the experiment of the sheet which records the position of the particle in every single experimental run. These two probabilities coincide even though they refer to different experimental set-ups, because these probabilities depend on the sample set $U$ only through the projector $\hat{P}_{U}$. For this reason, the projection op- erator $\hat{P}_{U}$ represents the proposition that the particle's position has been measured to lie in the set $U$, irrespective of the experimental procedure or the details of the measuring device. From the results of the YES-NO experiments we can unambiguously reconstruct all probabilistic information about a physical system. One is led, therefore, to the suggestion that the projection operators refer to the properties of quantum systems -as manifested in measurements- and that the structure of the lattice of projection operators represents the structure of potential quantum mechanical events. One speaks, therefore, for the quantum logic of quantum mechanical measurements.

In two-time measurements the situation is different. The measured probabilities are not functions of the single-time projectors. They depend instead on the properties of the measuring device and the way each sample set is resolved into minimum resolution sets. One may still perform two-time YES-NO experiments, by directing the particles through two successive slits and placing a detector behind the second slit to determine whether the particle has crossed through both slits or not. The expression (10) may be employed for the probabilities that are constructed in this experiment. But the filter measurements do not suffice to reconstruct the twotimes probability assignment to the physical quantity they represent; the physical probabilities for the two-time experiments are given by equation (22) and not by equation (10). There is no a priori physical reason that the probability that the particle will cross the slit will be the same with the probability obtained from the relative frequencies of the events within $U$ in the latter measurement. In single-time measurements they happen to be the same, but in two-time ones they are not.

In other words, the YES-NO experiments do not capture all physical information about two time measurements - the physical predictions depend strongly on the properties of the measuring devices. Hence the proposition that the particle is measured at time $t_{1}$ within the set $U_{1}$ and at time $t_{2}$ within the set $U_{2}$ is not universally represented by the ordered pair of projectors $\left(\hat{P}_{U_{1}}, \hat{P}_{U_{2}}\right)$. It is only represented by these projectors when the measuring device consists of two filters the first with a slit corresponding to $U_{1}$ and the second with a slit corresponding to $U_{2}$. There is, therefore, no universality in the representation of measurement outcomes by pairs of projection operators, with the consequence that the interpretation of two-time measurements in terms of quantum logic is not possible.

We may make, in fact, a stronger statement: even for single-time measurements the interpretation in terms of quantum logic is not possible. The proof involves reductio ad $a b$ surdum. We represent the single-time lattice of projection operators on a Hilbert space $H$ as $L(H)$ and assume that each measurement outcome may be uniquely represented by an element of $L(H)$-the converse need not be true. Two successive measurement outcomes should, therefore, be represented by a pair of elements of $L(H)$, hence an element of $L(H) \times L(H)$. We have showed that this is not the case. Hence there exists an error in our assumptions. The statement that two measurement outcomes are represented by a pair of arguments of $L(H)$ is a consequence of basic princi- 
ples of logical reasoning. Unless we assume that a two-time measurement does not correspond to two single-time measurements we are forced to conclude that the universal representation of measurement outcomes by projection operators is not valid even in single-time measurements. The reason it seems possible to do so, is because in single-time measurements the interference term $d$ of Eq. (12) always vanishes.

From the considerations above we are led to a distinction between the two different roles of the sample sets $U$ and the corresponding projectors - a distinction that is not usually made in probability theory. A sample set $U$ may represent a physical event, if the device can not distinguish between the elements of $U$. In that case $U$ refers to a concrete empirical fact. It may also represent a statement about the physical system, namely that an event has been found within the set $U$. The latter case, however, is not a representation of a physical fact. It is at the discretion of the experimentalist to choose the set $U$ that he will use for the sampling of its results. The physical probabilities should, therefore, be constructed with the first interpretation of the sample sets in mind. These probabilities then depend then on the construction of the physical apparatus and interaction with the measured system. They may also depend on the initial state of the measured system: ultra-fast neutrons, for instance, will leave a different trace on a recording material than slow ones. The crucial point is that quantum theory quantum theory distinguishes sharply between physical events and propositions about physical events, when such events refer to more than one moments of time.

\section{Second alternative: frequencies do not converge}

The conclusions above follow from the assumption that probabilities can be defined for multi-time measurements, namely that the sequences of relative frequencies converge. The second alternative has the advantage of rescuing the logical structure of quantum measurement, at the cost of making the probabilistic description incomplete. Its treatment of probabilities is essentially the same with that of the consistent histories approach. That is, we assume that the relative frequencies $\nu_{n}\left(U_{1}, t_{1} ; U_{2}, t_{2}\right) / n$ do not in general converge as $n \rightarrow \infty$. Hence the corresponding probabilities cannot be defined from the experimental data; again the resulting theory cannot be modelled by a classical stochastic process [8].

The hypothesis that two-times probabilities are not defined is not incompatible with the successful use of probability theory for single-time measurements. Probabilities are additive for single-time measurement - just as relative frequencies are- and there is no problem in that case to assume that the sequences $\nu_{n}\left(U_{1}, t_{1} ; U_{2}, t_{2}\right) / n$ converge. The same would be true for any sufficiently coarse-grained measurements, for which the interference term vanishes. One would be, therefore, led to the interpretation of the object $\left|\operatorname{Re} d\left(U_{1}, U_{j} ; U_{2}, U_{j}\right)\right|$ as a measure of the non-convergence of the sequence of relative frequencies. Probabilities would, therefore, be definable only for specific samplings of the measurement outcomes, such that the consistency condition
$\operatorname{Red}\left(U_{1}, U_{j} ; U_{2}, U_{j}\right)=0$ holds. This is, in fact, similar to the use of probabilities by the consistent histories approach probabilities are defined only for sufficiently coarse-grained partitions of the two-time sample space, such that the consistency condition is satisfied.

The important point is that the distinction between these two alternatives is not a 'mere' interpretational issue. The alternatives are empirically distinguishable. What is necessary for this purpose is a source of a weak particle beam that allows one to consider one particle at a time and two sheets that will detect (but not capture) the particles placed in a specific distance from the source. By measuring the particle's trace on the sheets and constructing the sequence $\left(x_{t_{1}}, x_{t_{2}}\right)_{n}$ of measurement outcomes in each experimental run-essentially the same thing we would do if the particles were described by classical probability theory- we may perform a statistical analysis to unambiguously determine whether the relative frequencies converge. We have showed elsewhere that the non-convergence of frequencies corresponding to $\left|\operatorname{Re} d\left(U_{1}, U_{j} ; U_{2}, U_{j}\right)\right|$ is beyond sampling errors in the set-up of such experiments [9]. For that purpose it is necessary that one considers a large number of runs, so that the statistical uncertainty in the determination of convergence (which goes with $1 / \sqrt{n}$ ) becomes very small. It is therefore to be expected that one may concretely identify the behaviour of relative frequencies in two-time measurements.

This experiment would enable one to state concretely the reason why quantum theory cannot be described by a classical stochastic process. If frequencies are found to converge, we would say that the failure is at the level of logic-multitime quantum measurements are so highly contextual that it is impossible to have a universal description in terms of logical propositions like the one assumed in quantum logic. If frequencies do not converge, then the failure is at the level of probabilities. Very simply quantum theory is not only a probability theory-probabilities cannot always be defined and on should seek for a different physical explanation for the mathematically natural 'measures' of Eq. (10), or equivalently for the complex-valued correlation functions [10]. Either way quantum measurement theory will be faced with a challenge.The first alternative implies that (multi-time) logical inferences from experimental data are typically unsound, and thus raises a demand for a consistent interpretational scheme for recovering some form of propositional logic for quantum events. The second alternative is less destructive, but it requires a deeper understanding of the mathematical relationship between the statistics of event frequencies and the standard Hilbert space formalism. More importantly, it brings about question of the physical origin of the 'instability' of quantum probabilities.

\section{Acknowledgements}

This work was supported by a Marie Curie Reintegration Grant of the European Commission and a research grant from the Empirikion Foundation. I would also like to thank N. Savvidou for many discussions and comments on that issue. 


\section{References}

[1] E. Nelson, Quantum Fluctuations (Princeton University Press, Princeton, 1985).

[2] R. Griffiths, Consistent Histories and the Interpretation of Quantum Mechanics, J. Stat. Phys. 36, 219 (1984); Consistent quantum theory (Cambridge University Press, 2003).

[3] R. Omnès, Logical Reformulation of Quantum Mechanics: I Foundations, J. Stat. Phys. 53, 893 (1988); The Interpretation of Quantum Mechanics, (Princeton University Press, Princeton, 1994); Consistent Interpretations of Quantum Mechanics, Rev. Mod. Phys. 64, 339 (1992).

[4] M. Gell-Mann and J.B. Hartle, Quantum mechanics in the Light of Quantum Cosmology, in: Complexity, Entropy and the Physics of Information, ed. by W.H. Zurek, (Addison Wesley, Reading, 1990); Classical Equations for Quantum Systems, Phys. Rev. D47, 3345 (1993).

[5] J.B. Hartle, Spacetime Quantum Mechanics and the Quan- tum Mechanics of Spacetime, in: Proceedings of the 1992 Les Houches School, Gravitation and Quantization (1993).

[6] C. Anastopoulos, On the relation between quantum mechanical probabilities and event frequencies, Ann. Phys. 313, 368 (2004).

[7] J.J. Jauch, Foundations of Quantum Mechanics (AddisonWesley, Reading, 1968).

[8] For a related interpretation of quantum probability, see: A. Khrennikov, Einstein and Bell, von Mises and Kolmogorov: Reality and Locality, Frequency and Probability, Found. Phys. 32, 1159 (2002).

[9] C. Anastopoulos, Experimental distinction of two alternatives for quantum probability, submitted to PRL (2004).

[10] For an interpretation in terms of geometric phases taken as irreducible parts of the quantum mechanical formalism, see: C. Anastopoulos, Quantum Theory without Hilbert Spaces, Found. Phys. 31, 1545 (2001); Quantum Processes on Phase Space, Ann. Phys. 303, 275 (2003). 